Mr. Hawkins exhibited as his last and best production, being both effective and cheap, a craniometer, consisting of a metal tube about six inches long, and a quarter of an inch inner diameter, to be laid horizon. tally across the top of the head: into each end of which horizontal tabe, a tube three inches long slides, and against the end of each of the sliding tubes is firmly fixed a vertical tube two inches long, open at both ends, and about an eighth of an inch internal diameter. Through the vertical tubes two wires slide, each seven inches long, having about an inch of the lower end bent at right angles to the remaining six inches of its length, the shorter of the two arms of the wire being terminated by an ivory ball a quarter of an inch in diameter. In order to keep the two balls in a line pointing towards each other, a groove is cut along the back of each sliding wire, and an elastic tongue is formed in the vertical tube to press into the groove, and serve the double purpose of a guide to prevent the wire turning around, and of a spring to allow of easy sliding motion, without liability of slipping with its own weight.

In using this instrument for taking the altitude of the head above the line passing through the orifices of the ears, the ivory balls are placed in the ears, and the horizontal tubes slidden down upon the vertical wires until the middle part touches the top of the head. The balls are then taken out of the ears, which the sliding of the horizontal tubes allows of being easily done, the instrument is laid down on a table, and the distance from the horizontal tube to the centres of the balls measured with a rule, or the wires may be graduated to show the measurement on inspection. By a similar application to the front or back of the head, the respective distances from the line may be obtained.

Mr. Hawkins did not recommend this craniometer as a substitute for the common calliper, for taking the distances of any two accessible points, for which purpose the common calliper is rather more convenient.

For taking curvatures, he showed a wire, from one-tenth to one-eighth of an inch diameter, made of pure grain tin, which is so pliable that it takes the form of any curve over which it is pressed, and by reason of its freedom from elasticity, retains the figure with sufficient firmness to be taken off the head and laid down on paper, where it may be held by laying a stick across the two ends, while a pencil is passed around inside the wire to delineate the curvature of the part measured.

He also exhibited a very simple instrument, by means of which curves may be taken upon paper immediately from a skull or cast held down upon the paper.

This instrument consists of a circular piece or disc of wood, about three inches diameter, and an inch thick in the middle, but thinned off to half an inch at the circum. ference, one side being flat. Near the circumference a metal tabe, one inch long and one-sixth of an inch diameter, is fixed, per. pendicular to and even with the flat side of the disc, but projecting from the uneven side; through this tube a short bit of pencil slides freely, and is pressed with force enough to mark on paper, by a spiral spring placed tightily on the tube, and bearing on the end of the pencil.

From the middle of the uneven side of the disc arises, perpendicular to the flat side, a cylindrical stem, five inches long and five. eighths of an inch diameter, having a groove along its whole length on the side towards the pencil: upon this stem a spring socket slides, carrying an arm projecting at right angles from the stem; the extremity of which arm is formed in to a knife-edge figure: this edge is always in a line with the axis of the pencil, being guided by a tongue slid. ing in the longitudinal groove of the stem.

It is obvious that the knife-edge end of the arm being passed around any part of a skull or cast held down upon paper, while the flat face of the dise is passed over the surface of the paper, the pencil will mark the outline passed over by the tracing arm.

Mr. Hawkins exhibited diagrams of two heads of active-minded persons, which he had measured at different periods; from which it was seen that one head had grown three-eighths of an inch in height in twenty. four years, between the ages of thirty and fifty-four; and the same head had acquired an eighth of an inch of additional height in fifteen years, from the ages of fifty-four to sixty-nine. The other head had gained near a quarter of an inch in hejght in twelve year's, from the ages of fifty-five to sixty. seven.

Mr. Hawkins concluded with offering to communicate, gratuitously, the results of his experience to any young man who would undertake the manufacture of these instru. ments for sale.

Mr. Deville recommended a flat tin strap, about three-eighths of an inch wide, and a twentieth of an inch thick, as preferable to the wire.

\section{OBSERVATIONS ON THE RESULT} OF VARICOSE OPERATIONS.

By Charles Clay, Surgeon, Manchester, Lecturer on Medical Jurisprudence.

In The Lancet of August, 1840, I directed the attention of the medical profession to the new operation, first proposed by $M$. Laugier, for the cure of varicose veins so prevalent amongst females.

In that communication I gave a case in which I had added some little improvements to the original, as recommended and prace 
tised by Laugier. In The Lancet of October following, I made some further observations, with a second illustrative case ; and $I$ recorded a third confirming the same views in your Journal of December in the same year, since which time other opportanities have occurred enabling me to test the value of this operation more satisfactorily, from which $I$ feel justified in concluding that it will be found a very valuable operation, fully realising all that can be expected from it. It is necessary, however, that the merits of this operation should be tested more generally by the profession, before it can be received as the best means of relief in such cases; it is with a view that it may become more generally known, and its merits more freely tested, that I make these additional remarks to my former papers. I have now operated in fourteen cases, in four of which I have had to operate on both Iegs; and when it is considered that two or three distinct operations have to be performed upon every leg so affected, it follows, then, that I have in the whole practised this operation near fifty times. Why I state this particularly, is to show how little is to be apprehended from the operation, as I have not had a single instance where any serious symp. toms have arisen from inflammation of the veins, although $I$ have performed it under the worst possible circumstances of constitution and age, also during every kind of weather, yet the whole have progressed well (at least with one or two exceptions $I$ shall hereafter allude to), varying as to the time of complete recovery, according to circum. stances connected with the constitution or previous habits of the patient.

Many as are the advantages of this operation, I wish to report those cases where, although they cannot be styled successful, yet the patients have been so far benefitted that the reader will conclude with me, that neither of them disprove the value of the means employed, when it is considered that out of fourteen cases two only were met with that did not fully realise the expectation, it must be allowed to be a very small proportion, and, in point of number, would have little weight against its general adoption, particularly, as I have before stated, when it can be shown that both the cases alluded to derived considerable benefit.

The first of these was a female, pregnant of the fifth child, having to stand ten hours daily at her employment in a cotton factory, of high temperature, and subjected to brutal treatment from her husband almost every day, with scarcely sufficient food, yet under all these very unfavourable circumstances the leg operated upon got well; but in about four mosths after, in consequence of violent kicks received from her husband on the same leg, the veins became again varicosed, but not in the immediate vicinity where the operations had been previously performed; but so far was she satisfied with the benefit she had derived from the operation, that she is quite willing to submit to a renewal of it on those veins now varicosed.

The second case, like the first, was miserably poor, and, therefore, destitute of comforts arising from good food and clothing, in addition to which she was fifty-six years of age, and had had eleven children; the varicose state of the veins had left the leg, but in consequence of one of the operations being too near the malleolus externus, a small portion of the bone got injured by the caustic, and an awkward sore followed, which is now slowly healing. Thus I have faith fully stated all the disadvantages that have arisen in fourteen cases and near fifty operations. A few observations resulting from the series of operations above-mentioned may not be out of place here: I find females bear the operation extremely well; indeed, as far as itself is concerned it is a mere trifle: the slow action of the Vienna paste afterwards requires both patience and determination. In young and healthy habits the sores will be quite healed in three weeks; but in those of advanced age, debilitated constitutions, and stinted means of subsistence, it will take four or five weeks. There will always be a tendency to inflammation along the course of the vein; but as I have stated before, no serious attack of that kind has been observed in $\mathrm{my}$ experience; all the inflammatory action 1 have met with easily gave way to emollient fomentations, with proper aftention to the bowels, and rest. It is necessary to caution the operatornot to make the incisions near the bones, avoiding particularly the malleolus externus and internus and the edge of the tibia; if the operation is in a properly-selected part, it is sure to do well; but the more muscular the better, and the less the tendons are interfered with the more satisfactory will be the result. I have always selected the most distended trunks of the veins for the incision, and where they have approached nearest the surface. The morning after the operation $I$ remove all the Vienna paste $I$ can from the wound, taking especial care not to disturb the portion next to the vein, and which has been in active operation; from the removal of the extra paste, the entire treatment consists in emollient dressings, poultices, fomentations, \&c., with attention to the bowels. In some irritable habits, $I$ have had occasion to give an opiate after the operation.

In nearly all the cases $I$ have had to treat there existed previously foul-conditioned and long-standing ulcers, which have rapidly healed, very many of them, before the cicatrix of the operation has been formed.

In all the cases, with the exceptions mentioned in this communication, there has been no tendency to any return of the disease, although some are ten or twelve months ago; from this $I$ am pretty confident the relief bj 
operation may be esteemed permanent; and that it is sufficiently successfal to merit further confidence is, I think, evident from the cases I have here written upon.

\section{REMARKABLE CASE OF ILEUS,}

FROM A CORD.LIKE PROLONGATION OF OMENTUM STRANGULATING A PORTION OF ILEUM OVER WHICH IT PASSED; DEATH FOLLOWED IN FORTY HOURS.

\section{To the Editor of ThE LANCET.}

Sir:-l beg to transmit to you the follow. ing interesting case which recently came under my care, and should you deem it of sufficient importance to merit publicity through the mediam of your widely-extended and truly valuable Periorlical, you will oblige me by its early insertion. I am, Sir, your most obedient servant,

Edinburgh, July 13, 1841 .

Robert Murray.

Mrs. Morris, ætat. 57, but from her very attenuated form and careworn visage appeared, at least, ten years older, of melancholic temperament, and costive habit, has given birth to nine children; three years ago had a violent attack of ileus, which obstinately resisted the most powerful enemata and other remedial means then employed for its relief. The medical attendants had given her up as a hopeless case, when, much to their astonishment, and no less so to the joy and satisfaction of her friends, the bowels opened spontaneously in the evening of the fourth day from the invasion of the attacks. Her convalescence was tardy and imperfect, and ever since she has, from time to time, suffered from repeated paroxysms of colic, more especially if she neglected taking aperient medicine, which she required to do, at least, thrice in the week. Soon after breakfast on the 30th of May last, symptoms of ileus again began to manifest themselves; these, however, were thought lightly of at first, but towards night they set in with augmented severity. Belonging to the humbler class of society, her friends did not seek medical assistance, until the disease had got thirty hours in advance; in the interim a dose of castor-oil was administered (her bowels had not been open for three days), which, however, was rejected as soon as swallowed. On the following day, at three, P.M., I was summoned to attend her. I found the poor creature in great bodily agony, and in a state of complete collapse; the pulse was gone at the wrist, and the heart's action extremely feeble; the temperature of the whole body was also much below the natural standard. The other symptoms were, stercoraceous vomiting, recurring at intervals of two or three minutes; acute pain of the abdomen, particularly a little to the zight of the umbilical region; it was, however, relieved to a cer. tain extent by pressure; the countenance indicated great suffering and anxiety; the eye appeared dull and glassy; the tongue was parched and covered with a brown fur, and the thirst insatiable; the respirations very hurried, and hiccup had commenced. From these symptoms, I at first suspected the woman to be labouring under strangulated hernia; I accordingly examined those regions where hernia usually occurs, but could not perceive the smallest trace of swelling or pain in either of them. I or. dered the following plan of treatment:-An enema composed of

Oil of turpentine, $\bar{j} j$;

Tincture of opium, $3 \mathrm{j}$;

Thin gruel, $0 \mathrm{j}$.

To be thrown up the rectum immediately, and repeated (omitting the laudanum) every hour, until full and free evacuations were obtained; cloths wrung out of warm turpen. tine to be applied to the abdomen, and bottles of hot water to the feet.

I saw her again in three hours, slight re. action had taken place; the pulse could be indistinctly felt at the wrist, small, intermit. ting, and thready. All the other symptoms were aggravated; the pain in the abdomen -now tympanitic-was excruciating and incessant, and very tender upon pressure. Neither of the injections could he thrown up, for, on attempting to do so, they returned instantly. Seeing that the case was utterly hopeless, and in order to render the scene less distressing, I ordered,

$$
\begin{aligned}
& \text { Calomel, gr. ij ; } \\
& \text { Opium, gr. j. }
\end{aligned}
$$

To be taken every hour. Twelve leeches to the abdomen. She got rapidly worse, and at two the next morning sunk.

Post-mortem Appearances.-Having ob. tained the friends' consent to make an examination of the abdomen and its contents, I proceeded to examine them thirty hours after death. The abdomen was much distended, and emitted a dull sound by percus. sion. On exposing the peritoneum investing the abdominal parietes, it was found to be a little more vascular than natural; the great omentum presented a similar appear. ance. Endeavouring to raise the latter, for the purpose of examining the small intes. tines, a powerful resistance was met with at its inferior margin and to the right side. I explored it with the utmost care, and found the resistance to arise from a prolongation of the great omentum, but differing from it in appearance and feel, being round, tendinous, firm, and inelastic. This tendjnous-looking cord was given off from the right inferior angle of the omentum, and proceeded downwards and a little outwards, and was reflected over the (right) rond ligament of the uterns, a bout three inches from the internal inguinal ring, where it became continuous with the pelvic portion of peritoneum. This abnormal structure, which 